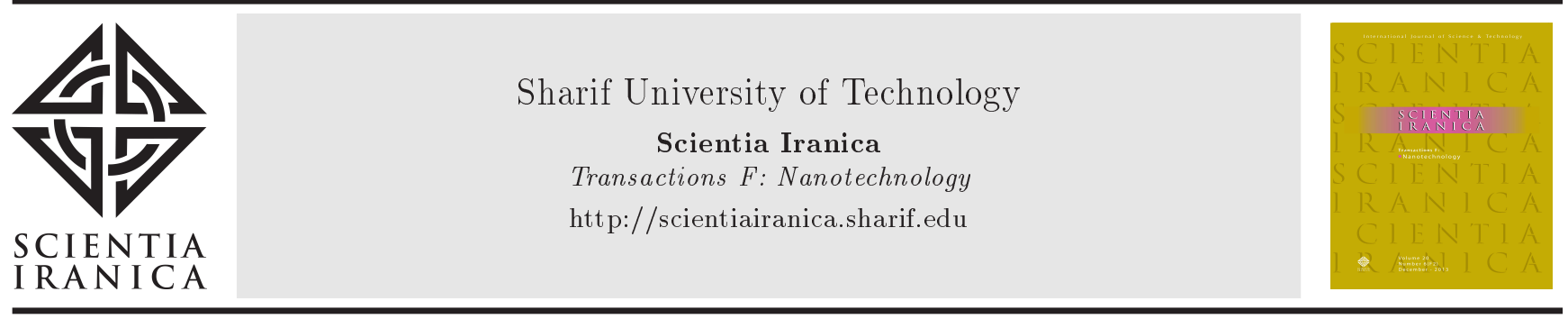

\title{
Consequences of activation energy and chemical reaction in radiative flow of tangent hyperbolic nanoliquid
}

\author{
S. Jabeen ${ }^{a, *}$, T. Hayat ${ }^{a, b}$, A. Alsaedi ${ }^{b}$, and M.Sh. Alhodaly \\ a. Department of Mathematics, Quaid-I-Azam University 45320, Islamabad 44000, Pakistan. \\ b. Nonlinear Analysis and Applied Mathematics (NAAM) Research Group, Department of Mathematics, Faculty of Science, King \\ Abdulaziz University, Jeddah 21589, Saudi Arabia.
}

Received 18 January 2019; received in revised form 7 March 2019; accepted 27 April 2019

\author{
KEYWORDS \\ Tangent hyperbolic \\ nanofluid; \\ Double stratification; \\ Joule heating; \\ Mixed convection; \\ Non-linear thermal \\ radiation; \\ Activation energy.
}

\begin{abstract}
This study explores mixed convection flow of tangent hyperbolic liquid over stretching sheet. Joule heating, double stratification, non-linear thermal radiation, Brownian motion, and thermophoresis were presented. The phenomenon of mass transfer was examined by activation energy along with binary chemical. Computations of convergent solutions were carried out for the nonlinear mathematical system. Graphical representation was employed to illustrate the outcome of sundry variables on velocity, temperature, and concentration of nanoparticles. Moreover, Nusselt number, coefficient of drag force, and mass transfer rate were examined. It was observed that velocity decreases at a larger Weissenberg number. Concentration of fluid was enhanced in the case of higher activation energy parameter.
\end{abstract}

(C) 2019 Sharif University of Technology. All rights reserved.

\section{Introduction}

Thermal radiation involves electromagnetic radiations transmitted due to the thermal motion of the fluid particles almost in all directions. The process of thermal radiation is observed in the heating of the bodies by diverse means such as solar light, fire, and radiator. Heat transfer with radiation has extensive industrial applications including atomic reactor security, boiler design, heat exchangers, power stations, and many propulsion equipment pieces and tools for missiles, aircraft, space automobiles, and satellites.

*. Corresponding author. Tel.: +92 5190642172 E-mail address: sumaira.jabeen@math.qau.edu.pk (S. Jabeen)

doi: $10.24200 /$ sci.2019.52726.2860
Radiative heat change in energy equation is measured by Rosseland calculation. Cortell [1] investigated nonlinear radiative heat transfer in flow by stretched surface. Properties of non-linear thermal radiation and cubic autocatalysis in nanofluid flow with rotational effects were described by Kumar et al. [2]. The effect of non-linear radiative flow on magneto-Burgers fluid with gyrotactic microorganisms was studied by Khan et al. [3]. Bhatti and Rashidi [4] developed a numerical solution to the problem of entropy generation minimization for radiative non-linear flow over a stretching sheet. Few recent efforts under this aspect can be observed in [5-7].

Examination of heat transfer via mixed convection has produced substantial interest in numerous fields of engineering and technology due to its applications to heat conversion in nuclear reactors, temperature variant atmospheric flow, electrical devices, 
and the flow path owing to density variation in a stream across a vertical direction through seasonal variation. Mixed convection is normally entitled through buoyancy force created by density and temperature variations. By Boussinesq's theory, equations of energy and momentum containing mixed convection term are highly coupled. Fluid flow with mixed convection generates a boundary layer close to the vertical plate. A sizeable literature on mixed convective flow is available now. Some representative contributions in this regard can be seen in [8-14].

Effects of mass transfers in fluid mixture are observed due to concentration variation of species existing in fluid. This variation occurs in a fluid when species move from a higher concentration region to a lower concentration region.

Further, the energy obtained by reactants before a chemical reaction is referred to as activation energy, which is also called the minimum energy required to initiate a reaction. Mass transfer phenomenon accompanied by chemical reaction along activation energy is frequently observed in several applications including chemical and geothermal engineering, mechanisms comprised of (oil, water) suspension, and food making. Recent contributions in this field are made only by few researchers [15-18].

Stratification is a process of developing layers through temperature and concentration variation or influence of different fluids. Through simultaneous effects of heat and mass transfer, it is important to analyze the effectiveness of thermal and solutal stratification concerned with stratification of fluid medium in view of heat convection and mass transfer because of convective motion of nanofluids. The study of mixed convection with double stratification is observed in diverse industrial and engineering applications. This phenomenon has a dominant role in polymer extrusion, in hydraulic flow of thermal fluids, geothermal reservoirs, volcanic flows, and geological systems. A number of studies have been conducted in this direction [19-22].

The current study discusses the influence of activation energy on double stratified flow of tangent hyperbolic nanofluid over a stretched surface. Modified Arrhenius function is used. Buongiorno model is considered, which emphasizes the novel features of thermophoresis and Brownian movement. In addition, mixed convection discloses the impact of buoyancy forces. Joule heating and nonlinear radiation are examined. Homotopy Analysis Method (HAM) is utilized for the development of convergent solutions [23-35]. Homotopic method appears to perform better in the following aspects. This method is not directly influenced by small or large parameters. Thus, HAM can be easily applied to weaker and strongly nonlinear problems. HAM is the unification of some other analytical methods in- cluding delta approximation, Adomian decomposition method, homotopy perturbation technique, and Lyapunov artificial parameter method. Explicit solutions to highly nonlinear problems can be calculated by HAM. It provides freedom to choose initial guesses and operators. Unlike other methods, HAM provides the simplest way to ensure the convergence of series solution.

\section{Modeling}

Unsteady flow of tangent hyperbolic nanofluid is examined here. Impermeable stretched sheet (at $y=0$ ) is accountable for fluid motion. Material density is assumed constant. Fluid confined the space $y>0$. Sheet is stretched with velocity $U_{w}(x)=a x$ in $x$ direction. The sheet has a constant temperature $\left(T_{w}\right)$ and constant concentration $\left(C_{w}\right)$, whereas $C_{\infty}$ and $T_{\infty}$ are fluid's ambient concentration and temperature, respectively. Brownian diffusion and thermophoresis are present. Magnetic fluid flows in the transverse direction (Figure 1). Effects of electric field are assumed negligible. Moreover, the influence of activation energy with binary chemical reaction will be detailed. Thermal radiation is taken to be non-linear. Viscous dissipation and heat generation/absorption are absent. Under these considerations, the velocity, temperature, and concentration expressions are follows $[4,17,35]$ :

$$
\begin{aligned}
& \frac{\partial u}{\partial x}+\frac{\partial v}{\partial y}=0 \\
& u \frac{\partial u}{\partial x}+v \frac{\partial u}{\partial y}=v(1-\tilde{n}) \frac{\partial^{2} u}{\partial y^{2}}+\sqrt{2} v \Gamma \tilde{n} \frac{\partial u}{\partial y} \frac{\partial^{2} u}{\partial y^{2}} \\
& \quad-\sigma \frac{B_{0}^{2}}{\rho_{f}} u+g\left[\beta_{T}\left(T-T_{\infty}\right)+\beta_{C}\left(C-C_{\infty}\right)\right]
\end{aligned}
$$

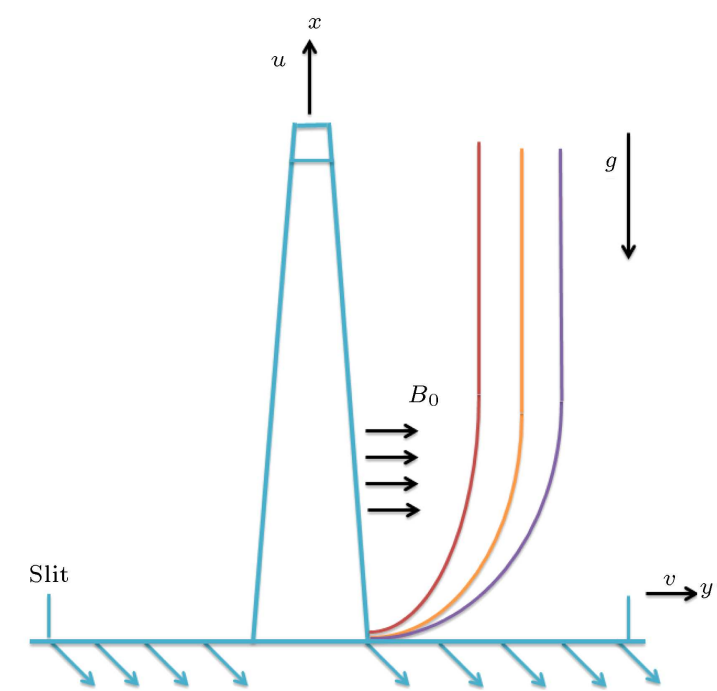

Figure 1. Physical model. 


$$
\begin{aligned}
\left(u \frac{\partial T}{\partial x}\right. & \left.+v \frac{\partial T}{\partial y}\right)=\frac{\breve{k}}{\left(\rho c_{p}\right)_{f}}\left(\frac{\partial^{2} T}{\partial y^{2}}\right) \\
& +\tau D_{B}\left(\frac{\partial T}{\partial y}\right)\left(\frac{\partial C}{\partial y}\right)+\tau \frac{D_{T}}{T_{\infty}}\left(\frac{\partial T}{\partial y}\right)^{2} \\
& \left.+\sigma \frac{B_{0}^{2}}{\left(\rho c_{p}\right)_{f}} u^{2}-\frac{1}{\left(\rho c_{p}\right)_{f}} \frac{\partial \breve{q}_{r}}{\partial y}\right) \\
u \frac{\partial C}{\partial x}+ & v \frac{\partial C}{\partial y}=D_{B}\left(\frac{\partial^{2} C}{\partial y^{2}}\right)+\frac{D_{T}}{T_{\infty}}\left(\frac{\partial^{2} T}{\partial y^{2}}\right) \\
- & K_{r}^{2}\left(C-C_{\infty}\right)\left[\frac{T}{T_{\infty}}\right]^{n} \exp \left[\frac{-E_{a}}{k_{1} T}\right] .
\end{aligned}
$$

The relevant boundary conditions are:

$$
\begin{aligned}
& u=U_{w}(x)=a x, \quad v=0, \\
& T=T_{w}=T_{0}+\breve{a} x, \quad C=C_{w}=C_{0}+\breve{e} x, \\
& \text { at } y=0 \text {, } \\
& u \rightarrow 0, \quad T \rightarrow T_{\infty}=T_{0}+\breve{b} x, \quad C \rightarrow C_{\infty}=C_{0}+\breve{d} x \\
& \text { at } y \rightarrow \infty \text {. }
\end{aligned}
$$

Here, $u$ and $v$ depict the velocity components parallel to $x$ - and $y$-axes, $\Gamma$ material constant, $\tilde{n}$ power law index, $v$ kinematic viscosity, $\rho$ fluid density, $c_{p}$ specific heat, $\mu$ the dynamic viscosity, $D_{T}$ the thermophoresis coefficient, $U_{w}$ the stretching velocity, $D_{B}$ the Brownian coefficient, $\tau$ the heat capacity of nanoparticles, $\sigma$ the electrical conductivity, $T_{0}$ the reference temperature, $\bar{k}$ the thermal conductivity, $\beta_{T}$ thermal expansion coefficient, $\beta_{C}$ concentration expansion coefficient, and $(\breve{a}, \breve{b}, \breve{e}, \bar{d})$ the dimensional constants. Further, $T_{w}$ and $C_{w}$ are the constant temperature and solute concentration near the surface, while $T_{\infty}$ and $C_{\infty}$ represent ambient temperature and concentration. Term $\left[\frac{T}{T_{\infty}}\right]^{n} \exp \left[\frac{-E_{a}}{k_{1} T}\right]$ in Eq. (4) is referred to as the modified Arrhenius function. In addition, $k_{1}=8.61 \times$ $10^{-5} \mathrm{eV} / \mathrm{K}$ represents the Boltzmann constant, $n$ is the dimensionless constant or rate constant in the range $-1<n<1, K_{r}$ is the chemical reaction parameter, and $E_{a}$ is the activation energy. Considering the following transformations:

$$
\begin{array}{ll}
\psi=\sqrt{a v} x f(\eta), & \eta=\sqrt{\frac{a}{v}} y, \\
\theta(\eta)=\frac{T-T_{\infty}}{T_{w}-T_{0}}, & \phi(\eta)=\frac{C-C_{\infty}}{C_{w}-C_{0}},
\end{array}
$$

velocity components are defined in terms of stream function $(\psi)$ by $v=-\frac{\partial \psi}{\partial x}$ and $u=\frac{\partial \psi}{\partial y}$. Now:

$$
u=a x f^{\prime}(\eta), \quad v=-\sqrt{a v} f(\eta) .
$$

Radiative heat flux through the Rosseland approximation yields:

$$
\begin{aligned}
\frac{\partial \breve{q}_{r}}{\partial y} & =\frac{-4 \sigma^{*}}{3 k^{*}} \frac{\partial}{\partial y}\left(\frac{\partial T^{4}}{\partial y}\right) \\
& =\frac{-16 \sigma^{*}}{3 k^{*}}\left[T^{3} \frac{\partial^{2} T}{\partial y^{2}}+3 T^{2}\left(\frac{\partial T}{\partial y}\right)^{2}\right],
\end{aligned}
$$

where $\sigma^{*}$ and $k^{*}$ represent Stefan-Boltzmann constant and mean absorption coefficient. Based on Eq. (9), Eq. (3) becomes:

$$
\begin{aligned}
& \left(u \frac{\partial T}{\partial x}+v \frac{\partial T}{\partial y}\right)=\frac{\breve{k}}{\left(\rho c_{p}\right)_{f}}\left(\frac{\partial^{2} T}{\partial y^{2}}\right) \\
& +\tau D_{B}\left(\frac{\partial T}{\partial y}\right)\left(\frac{\partial C}{\partial y}\right)+\tau \frac{D_{T}}{T_{\infty}}\left(\frac{\partial T}{\partial y}\right)^{2}+\sigma \frac{B_{0}^{2}}{\rho} u^{2} \\
& \left.\quad+\frac{1}{\left(\rho c_{p}\right)_{f}} \frac{16 \sigma^{*}}{3 k^{*}}\left[T^{3} \frac{\partial^{2} T}{\partial y^{2}}+3 T^{2}\left(\frac{\partial T}{\partial y}\right)^{2}\right]\right)
\end{aligned}
$$

Continuity expression is verified, and Eqs. (2) to (10) yield:

$$
\begin{gathered}
(1-\tilde{n}) f^{\prime \prime \prime}+f f^{\prime \prime}-\left(f^{\prime}\right)^{2}+\tilde{n} \mathrm{We} f^{\prime \prime} f^{\prime \prime \prime}-M^{2} f^{\prime} \\
+\lambda\left[\theta+N^{*} \phi\right]=0, \\
\theta^{\prime \prime}-\operatorname{Pr} S_{\theta} f^{\prime}+\operatorname{Pr}\left(f \theta^{\prime}-f^{\prime} \theta\right)+\operatorname{Pr} N_{b} \theta^{\prime} \phi^{\prime}+\operatorname{Pr} N_{t}\left(\theta^{\prime}\right)^{2} \\
+M E c^{2}\left(f^{\prime}\right)^{2}+\frac{4}{3} R_{d}\left\{\theta^{\prime \prime}\left[\theta\left(\theta_{w}-\theta_{0}\right)+1\right]^{3}\right. \\
\left.\left.+3\left(\theta^{\prime}\right)^{2}\left(\theta_{w}-\theta_{0}\right)\left[\theta\left(\theta_{w}-\theta_{0}\right)+1\right]^{2}\right\}=0\right), \\
\phi^{\prime \prime}+\frac{N_{t}}{N_{b}} \theta^{\prime \prime}+S c\left(f \phi^{\prime}-f^{\prime} \phi\right)-\operatorname{Sc} S_{\phi} f^{\prime} \\
-A^{\prime} \operatorname{Sc}\left[1+\theta\left(\theta_{w}-\theta_{0}\right)\right]^{n} \exp \left[\frac{-\stackrel{\circ}{1+\theta}}{\left.\theta_{w}-\theta_{0}\right)}\right]=0 \\
f^{\prime}(\eta)=1, \quad \quad f(\eta)=0, \quad \theta(\eta)=1-S_{\theta}, \\
\phi(\eta)=1-S_{\phi} \quad \text { at } \eta=0, \\
f^{\prime}(\eta) \rightarrow 0, \quad \theta(\eta) \rightarrow 0, \quad \phi(\eta) \rightarrow 0 \\
\text { at } \quad \eta \rightarrow \infty, \quad(1) \quad
\end{gathered}
$$

where We denotes the Weissenberg number, $M$ the magnetic parameter, $N^{*}$ the local buoyancy parameter, 
$\lambda$ the mixed convection parameter, $\operatorname{Pr}$ the Prandtl number, $S_{\theta}$ the thermal stratified parameter, $S_{\phi}$ the solutal stratified parameter, Sc the Schmidt number, $\stackrel{\circ}{E}$ dimensionless activation energy, and $A^{\prime}$ the dimensionless chemical reaction parameter. Furthermore, Ec, $R_{d},\left(\theta_{w}, \theta_{0}\right), N_{b}, N_{t},\left(\mathrm{Gr}, \mathrm{Gr}^{*}\right)$ are the Eckert number, radiation parameter, temperature ratio parameter, Brownian motion parameter, thermophoresis parameter, and the Grashof number (temperature and concentration), respectively. These parameters are defined as follows:

$$
\begin{array}{ll}
\mathrm{We}=\sqrt{\frac{2 a^{3}}{v}} \Gamma x, & M^{2}=\frac{\sigma B_{0}^{2}}{\rho a}, \\
\mathrm{Gr}=\frac{g \beta_{T}\left(T_{w}-T_{0}\right) x^{3}}{v^{2}}, & \lambda=\frac{\mathrm{Gr}}{\mathrm{Re}_{x}^{2}}, \\
\mathrm{Gr}^{*}=\frac{g \beta_{C}\left(C_{w}-C_{0}\right) x^{3}}{v^{2}}, & N^{*}=\frac{\mathrm{Gr}^{*}}{\mathrm{Gr}}, \\
R_{d}=\frac{4 \sigma^{*} T_{\infty}^{3}}{\breve{k} k^{*}}, \quad S_{\theta}=\frac{\breve{b}}{\breve{a}}, & \mathrm{Ec}=\frac{U_{w}^{2}}{\left(T_{w}-T_{0}\right) c_{p}} \\
\theta_{w}=\frac{T_{w}}{T_{\infty}}, \quad \theta_{0}=\frac{T_{0}}{T_{\infty}}, & \mathrm{Sc}=\frac{v}{D_{B}}, \\
\operatorname{Pr}=\frac{v}{\alpha}, \quad N_{b}=\frac{\tau D_{B}}{v} \breve{e} x, & N_{t}=\frac{\tau D_{T}}{v T_{\infty}} \breve{a} x, \\
S_{\phi}=\frac{\breve{d}}{\bar{e}}, \quad \stackrel{\circ}{E}^{2}=\frac{E_{a}}{k_{1} T_{\infty}}, & A^{\prime}=\frac{K_{r}^{2}}{a} .
\end{array}
$$

Coefficient of drag force $\left(C_{f x}\right)$, heat transfer rate $\left(\mathrm{Nu}_{x}\right)$, and mass transfer rate $\left(\mathrm{Sh}_{x}\right)$ are described as follows [30]:

$$
\begin{aligned}
C_{f x} & =\frac{\breve{\tau}_{w}}{\frac{1}{2} \rho U_{w}^{2} x}, \quad \mathrm{Nu}_{x}=\frac{x \breve{q}_{w}}{\breve{k}\left(T_{w}-T_{0}\right)}, \\
\mathrm{Sh}_{x} & =\frac{x \breve{q}_{m}}{D_{B}\left(C_{w}-C_{0}\right)},
\end{aligned}
$$

where:

$$
\begin{aligned}
& \breve{\tau}_{w}=\mu\left[(1-\tilde{n}) \frac{\partial u}{\partial y}+\frac{\Gamma \tilde{n}}{\sqrt{2}}\left(\frac{\partial u}{\partial y}\right)^{2}\right]_{y=0}, \\
& \breve{q}_{w}=-\breve{k}\left\{\left[1+T^{3} \frac{16 \sigma^{*}}{3 \breve{k} k^{*}}\right]\left(\frac{\partial T}{\partial y}\right)\right\}_{y=0}, \\
& \breve{q}_{m}=-D_{B}\left(\frac{\partial C}{\partial y}\right)_{y=0} .
\end{aligned}
$$

In the non-dimensional form, we obtain:

$$
\begin{aligned}
\operatorname{Re}_{x}^{\frac{1}{2}} C_{f x} & =(1-\tilde{n}) f^{\prime \prime}(0)+\mathrm{We} \frac{\tilde{n}}{2}\left(f^{\prime \prime}(0)\right)^{2} \\
\operatorname{Re}_{x}^{\frac{1}{2}} \mathrm{Nu}_{x} & =-\theta^{\prime}(0)\left\{1+R_{d}\left[1+\left(\theta_{w}-\theta_{0}\right) \theta(0)\right]^{3}\right\} \\
\operatorname{Re}_{x}^{\frac{1}{2}} \operatorname{Sh}_{x} & =-\phi^{\prime}(0)
\end{aligned}
$$

in which $\operatorname{Re}_{x}=\frac{U_{w} x}{v}$ is the Reynolds number.

\section{Solution methodology}

Series solution of the above-mentioned system is obtained by the homotopic technique. Initial guesses $\left(f_{0}, \theta_{0}, \phi_{0}\right)$ and associated linear operators $\left(\widetilde{\mathbf{L}}_{f}, \breve{\mathbf{L}}_{\theta}, \widetilde{\mathbf{L}}_{\phi}\right)$ are as follows:

$$
\begin{aligned}
& f_{0}(\eta)=1-e^{\eta} \\
& \theta_{0}(\eta)=\left(1-S_{\theta}\right) e^{-\eta} \\
& \phi_{0}(\eta)=\left(1-S_{\phi}\right) e^{-\eta} \\
& \breve{\mathbf{L}}_{f}(\eta)=\frac{d^{3} f}{d \eta^{3}}-\frac{d f}{d \eta} \\
& \breve{\mathbf{L}}_{\theta}(\eta)=\frac{d^{2} \theta}{d \eta^{2}}-\theta \\
& \breve{\mathbf{L}}_{\phi}(\eta)=\frac{d^{2} \phi}{d \eta^{2}}-\phi, \\
& \breve{\mathbf{L}}_{f}\left[\tilde{X}_{1}+\tilde{X}_{2} e^{\eta}+\tilde{X}_{3} e^{-\eta}\right]=0 \\
& \breve{\mathbf{L}}_{\theta}\left[\tilde{X}_{4} e^{\eta}+\tilde{X}_{5} e^{-\eta}\right]=0 \\
& \breve{\mathbf{L}}_{\phi}\left[\tilde{X}_{6} e^{\eta}+\tilde{X}_{7} e^{-\eta}\right]=0
\end{aligned}
$$

where $\tilde{X}_{i}(i=1-7)$ represents the arbitrary constants.

\section{Convergence analysis}

Homotopy analysis technique is helpful to find convergent series solutions and provides a chance to sketch the region of convergence. This region can be controlled by setting appropriate values of $\hbar_{f}, \hbar_{\theta}$, and $\hbar_{\phi}$. Figure 2 displays the h-curves. It is analyzed that the ranges of parameters $\hbar_{f}, \hbar_{\theta}$, and $\hbar_{\phi}$ are $-1.5 \leq \hbar_{f} \leq$ $-0.5,-1.3 \leq \hbar_{\theta} \leq-0.4$, and $-1.4 \leq \hbar_{\phi} \leq-0.7$. Numerically obtained solution convergence is presented in Table 1. Here, the 20th order approximation is enough for momentum equation, while energy and concentration equations converge at the 25 th order approximation. 
Table 1. Convergence of HAM solutions when $\tilde{n}=A^{\prime}=0.1=$ We, $\lambda=0.3=M=$ Sc, $S_{\theta}=0.3=S_{\phi}$, $\operatorname{Pr}=1.5, R_{d}=0.5, \mathrm{Ec}=1.3=\stackrel{\circ}{E}, \mathrm{Sc}=0.8, N^{*}=1$, $N_{b}=0.1=N_{t}, \theta_{w}=1.1$, and $\theta_{0}=1.2$.

\begin{tabular}{cccc}
\hline $\begin{array}{c}\text { Order of } \\
\text { approximations }\end{array}$ & $-\boldsymbol{f}^{\prime \prime}(\mathbf{0})$ & $-\boldsymbol{\theta}^{\prime}(\mathbf{0})$ & $\boldsymbol{\phi}^{\prime}(\mathbf{0})$ \\
\hline 1 & 0.87717 & 1.0384 & 0.074223 \\
2 & 0.88392 & 1.1535 & 0.075501 \\
3 & 0.87568 & 1.1784 & 0.069273 \\
4 & 0.86657 & 1.1822 & 0.065361 \\
6 & 0.86657 & 1.1830 & 0.065025 \\
12 & 0.86901 & 1.1795 & 0.064510 \\
15 & 0.86902 & 1.1798 & 0.064469 \\
20 & 0.86903 & 1.1793 & 0.064242 \\
25 & 0.86903 & 1.1794 & 0.064303 \\
30 & 0.86903 & 1.1794 & 0.064303 \\
50 & 0.86903 & 1.1794 & 0.064303 \\
\hline
\end{tabular}

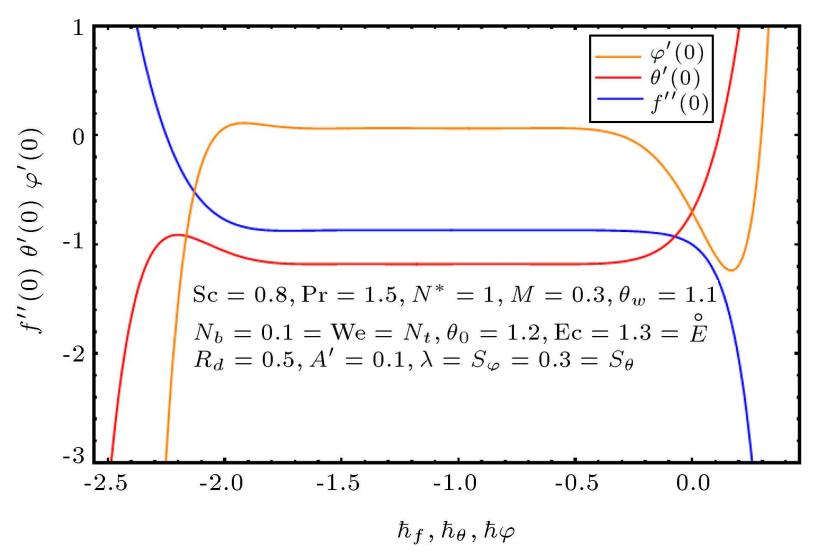

Figure 2. $\hbar$-plots in view of $f^{\prime \prime}(0), \theta^{\prime}(0)$, and $\phi^{\prime}(0)$.

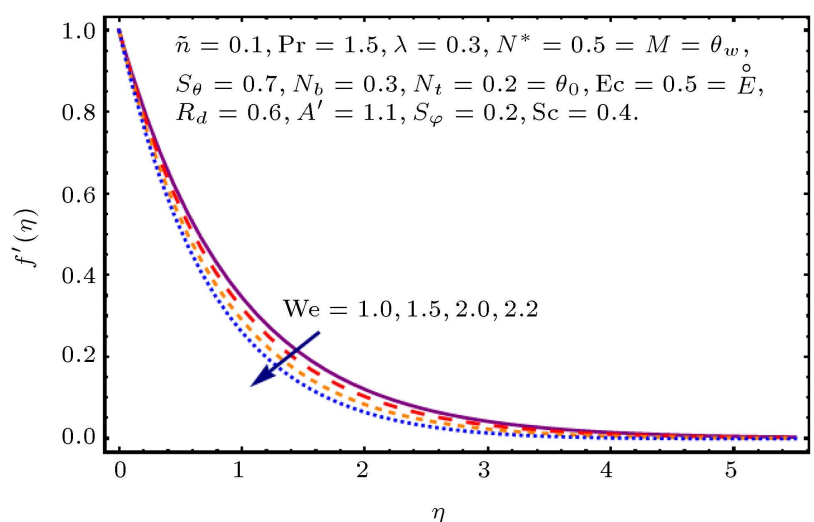

Figure 3. $f^{\prime}(\eta)$ via We.

\section{Discussion}

Velocity, temperature, and concentration are discussed in Figures 3-16. Figure 3 predicts Weissenberg number, We, variation on $f^{\prime}(\eta)$. Clearly, velocity and momentum layer have been found to be low through We. In fact, the Weissenberg number is the ratio of relaxation

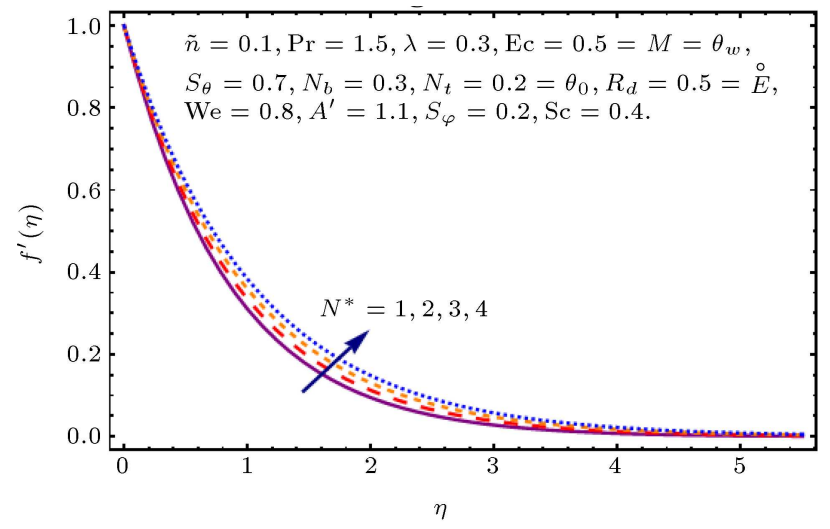

Figure 4. $f^{\prime}(\eta)$ via $N^{*}$.

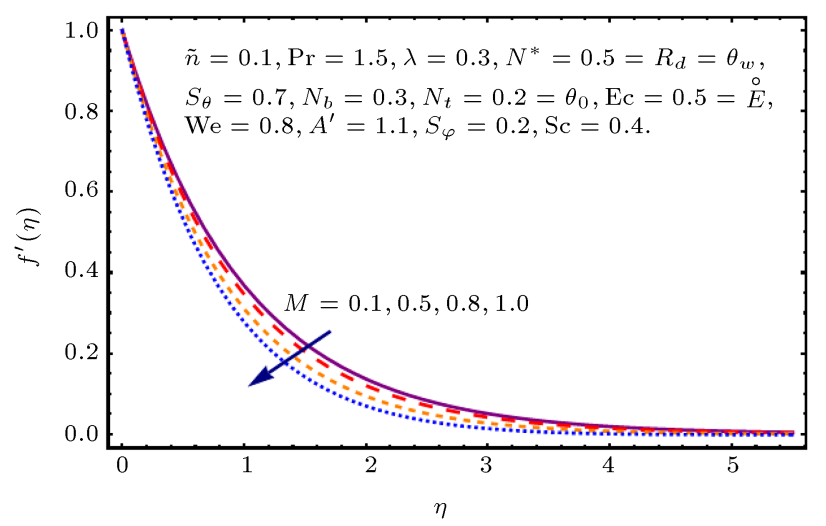

Figure 5. $f^{\prime}(\eta)$ via $M$.

time to particular time of fluid. Hence, for more relaxation time, the fluid thickness increases and, consequently, there is a reduction in fluid velocity. Figure 4 indicates the variation of velocity $f^{\prime}(\eta)$ by increasing local buoyancy parameter $N^{*}$. Clearly, velocity and related layer thickness are enhanced through larger $N^{*}$. Figure 5 illustrates the impact of magnetic parameter $M$ on $f^{\prime}(\eta)$. A rise in $M$ leads to a decrease in velocity field $f^{\prime}(\eta)$. Obviously, larger magnetic parameter $M$ increases Lorentz force (also termed as resistive force), which opposes fluid movement and, thus, velocity reduces. The influence of mixed convection parameter $\lambda$ is depicted in Figure 6. Velocity is enhanced through $\lambda$. Higher values of mixed convection enhance buoyancy forces; thus, velocity and corresponding momentum layer increase.

Figure 7 explores the variations of temperature field for magnetic parameter, $M$. In addition, temperature increases in the case of a larger magnetic parameter. Figure 8 demonstrates the enhancement of temperature and thermal layer at higher Eckert number, Ec. Graphical illustration of $N_{b}$ for $\theta(\eta)$ is presented in Figure 9. Temperature rises in response to larger variations of $N_{b}$, since the motion of fluid particle is unsystematic in the reaction of higher Brownian motion parameter that leads to an increase in heat 


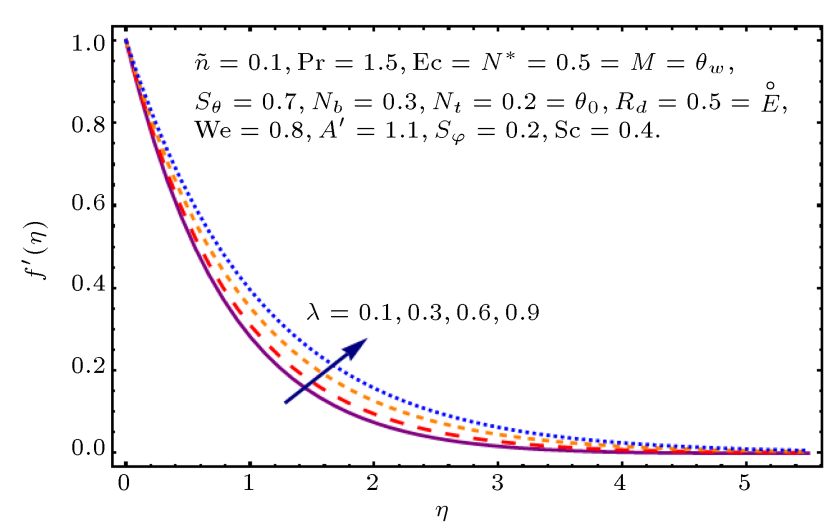

Figure 6. $f^{\prime}(\eta)$ via $\lambda$.

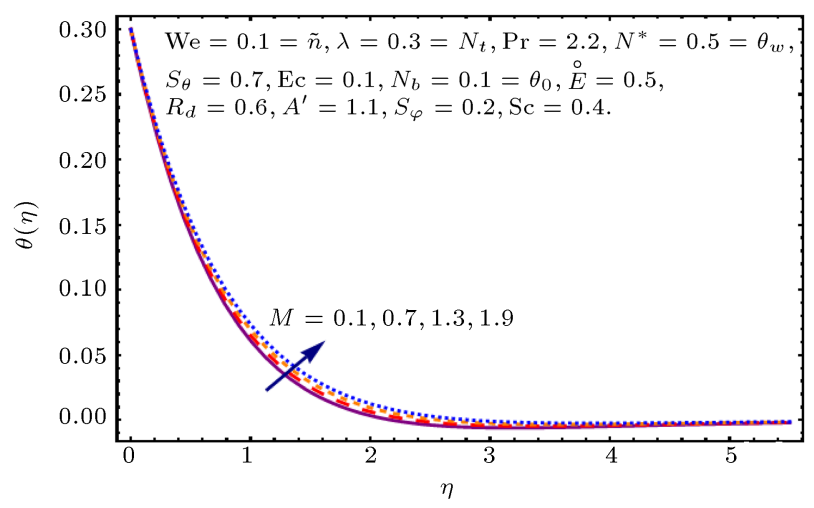

Figure 7. $M$ impact on $\theta(\eta)$.

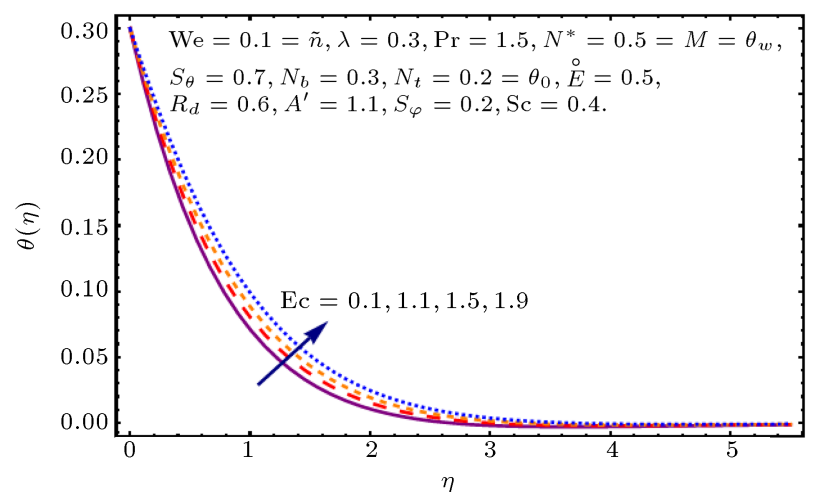

Figure 8. Ec impact on $\theta(\eta)$.

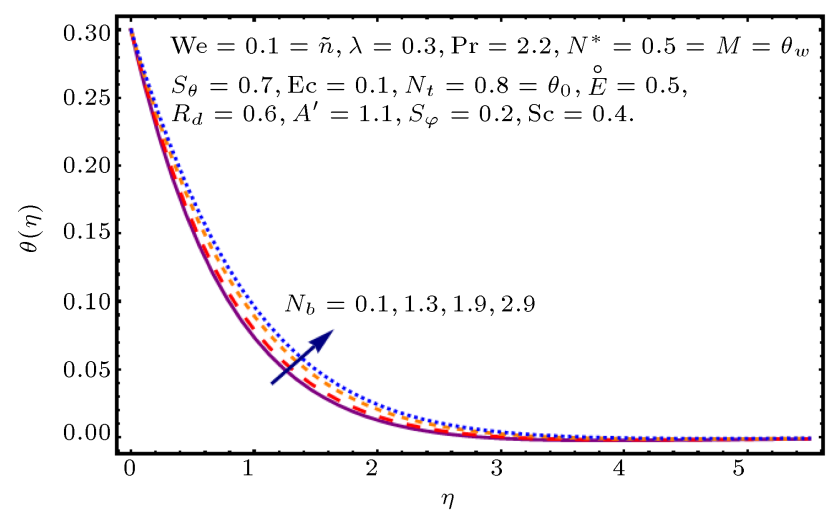

Figure 9. $N_{b}$ impact on $\theta(\eta)$.

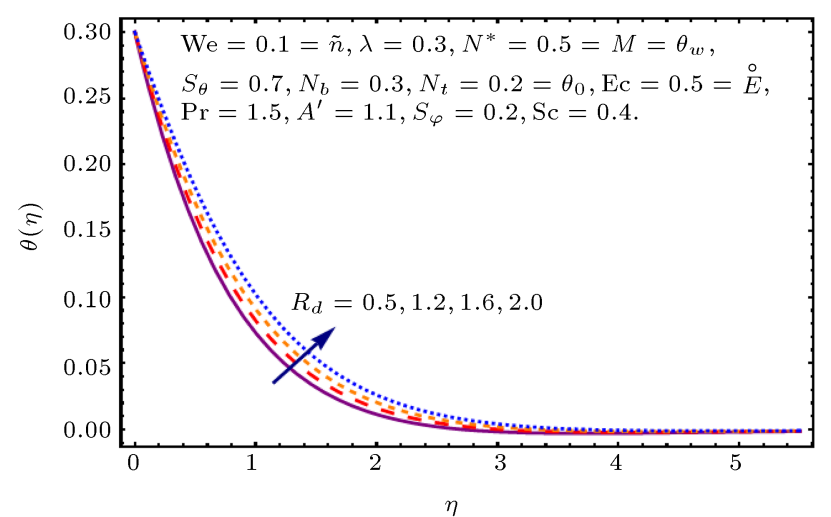

Figure 10. $R_{d}$ impact on $\theta(\eta)$.

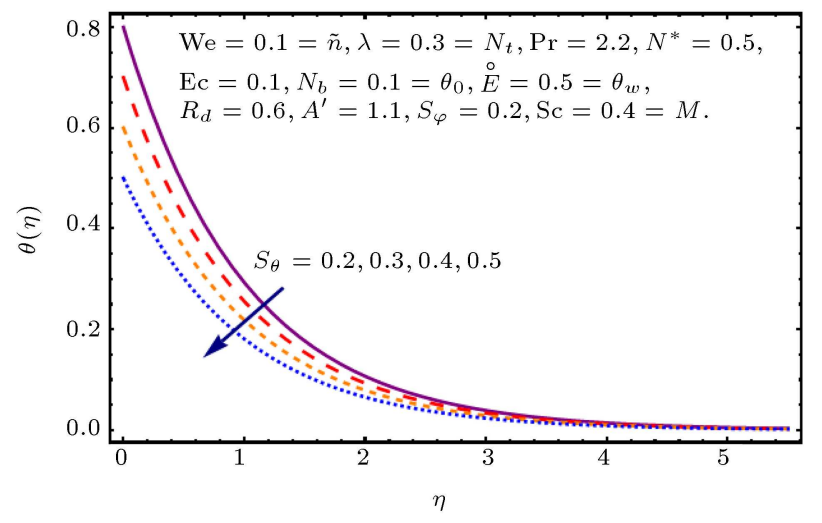

Figure 11. $S_{\theta}$ impact on $\theta(\eta)$.

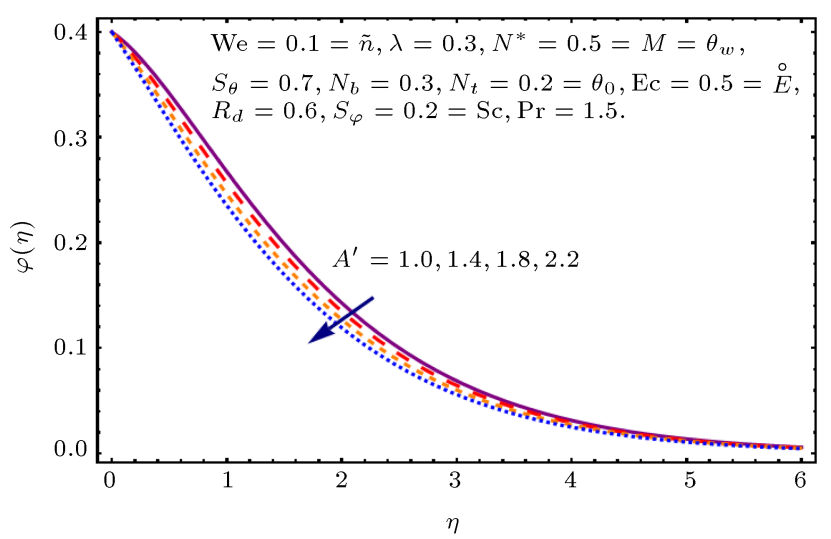

Figure 12. $A^{\prime}$ effect on $\phi(\eta)$.

production. Figure 10 explores temperature variation for radiation parameter, $R_{d}$. Larger $R_{d}$ gives rise to a higher rate of heat transfer since heat production enhances the radiation process. Impact of thermal stratified variable, $S_{\theta}$, on temperature, $\theta(\eta)$, is reflected in Figure 11. Thermal field decreases with an increase in $S_{\theta}$ as the difference in temperature decreases gradually between the ambient temperature, $T_{\infty}$, and surface temperature, $T_{w}$.

Figure 12 clarifies the outcome of $A^{\prime}$ on concentration of nanoparticles. As predicted, the decline 


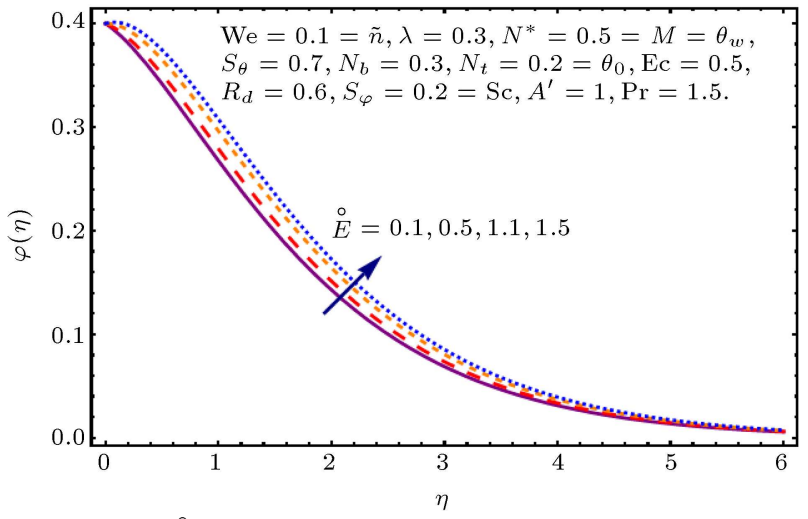

Figure 13. $\stackrel{\circ}{E}$ effect on $\phi(\eta)$.

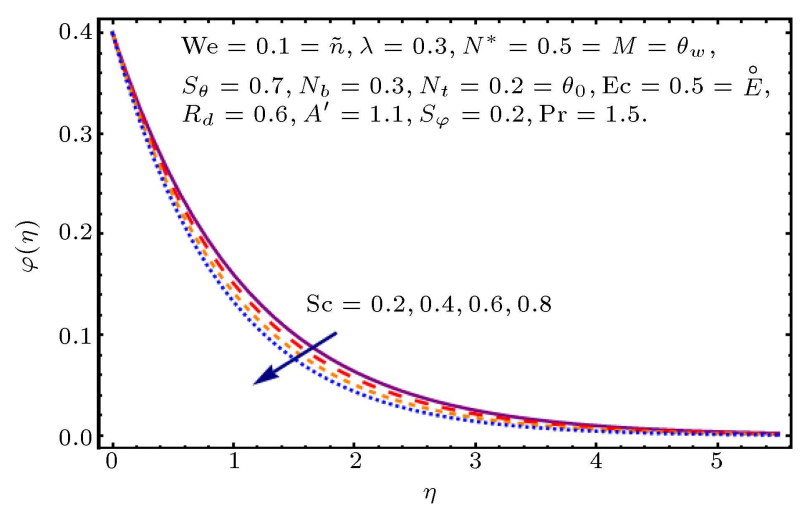

Figure 14. Sc effect on $\phi(\eta)$.

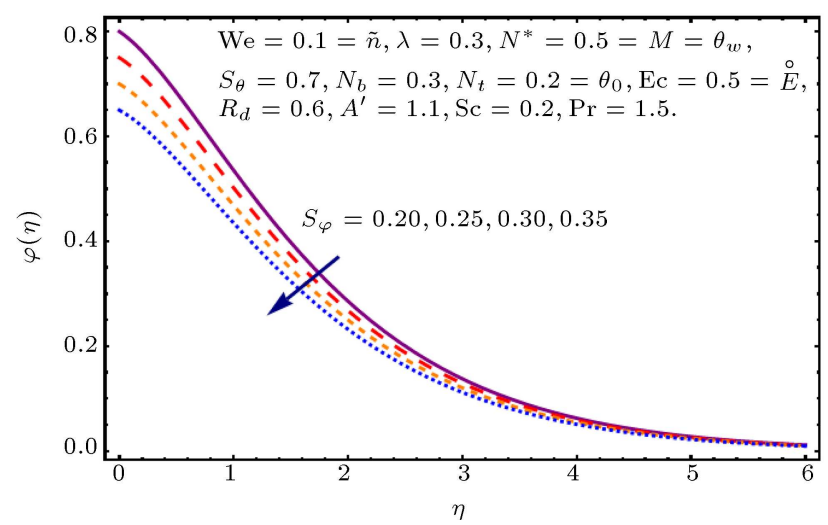

Figure 15. $S_{\phi}$ effect on $\phi(\eta)$.

in nanoparticle concentration is noticed for larger $A^{\prime}$. Variations of activation energy, ${ }^{\circ}$, in nanoparticles' concentration are shown in Figure 13. As expected, concentration is enhanced through $\stackrel{\circ}{E}$. Outcome of Schmidt number, Sc, on $\phi(\eta)$ is displayed in Figure 14. Herein, concentration decreases for higher Sc. Analysis of concentration stratification parameter, $S_{\phi}$, on $\phi(\eta)$ is displayed in Figure 15. It appears that larger values of $S_{\phi}$ correspond to lower nanoparticle concentration $\phi(\eta)$. Figure 16 demonstrates a marginal increase in the nanoparticle concentration when thermophoresis parameter $N_{t}$ varies from $N_{t}=0.2$ to $N_{t}=0.35$.

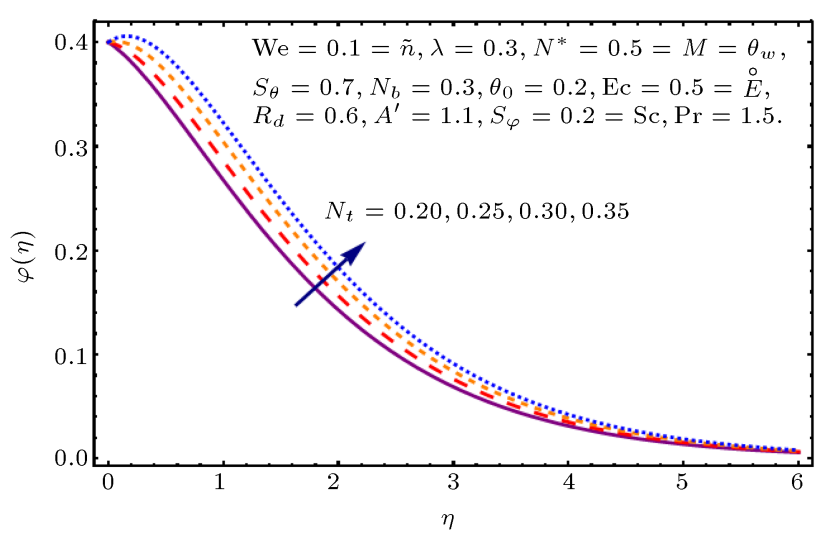

Figure 16. $\phi(\eta)$ via $N_{t}$.

Table 2. Numerical description of skin friction coefficient.

\begin{tabular}{ccccccc}
\hline$\tilde{\boldsymbol{n}}$ & $\mathbf{W e}$ & $\boldsymbol{M}$ & $\boldsymbol{\lambda}$ & $\boldsymbol{N}^{*}$ & $\stackrel{\circ}{\boldsymbol{E}}$ & $-\mathbf{R e}_{\boldsymbol{x}}^{\frac{1}{2}} \boldsymbol{C}_{\boldsymbol{f} \boldsymbol{x}}$ \\
\hline 0.1 & 0.1 & 0.3 & 0.3 & 1 & 1.3 & 0.8688595 \\
0.2 & & & & & & 0.9131529 \\
0.3 & & & & & & 0.9673078 \\
0.1 & 0.2 & 0.3 & 0.3 & 1 & 1.3 & 0.8701973 \\
& 0.3 & & & & & 0.8759865 \\
& 0.4 & & & & & 0.8783699 \\
0.1 & 0.1 & 0.4 & 0.3 & 1 & 1.3 & 0.9030014 \\
& & 0.5 & & & & 0.9458779 \\
& & 0.6 & & & & 0.9977259 \\
0.1 & 0.1 & 0.3 & 0.4 & 1 & 1.3 & 0.8039160 \\
& & & 0.5 & & & 0.7507146 \\
& & & 0.6 & & & 0.7290100 \\
0.1 & 0.1 & 0.3 & 0.3 & 1.2 & 1.3 & 0.4763013 \\
& & & & 1.3 & & 0.5473585 \\
& & & & 1.4 & & 0.6347946 \\
0.1 & 0.1 & 0.3 & 0.3 & 1 & 1.4 & 0.6814829 \\
& & & & & 1.5 & 0.5666427 \\
& & & & & 1.6 & 0.5298977 \\
\hline
\end{tabular}

Effects of various embedded parameters on skinfriction coefficient, Nusselt number, and Sherwood number are demonstrated in Tables 2-4. Table 2 declares that the coefficient of drag force is affected by mixed convection parameter, $\lambda$, and non-dimensional activation energy, $\stackrel{\circ}{E}$. The coefficient of drag force is decreased by these parameters. Skin-friction coefficient shows an increasing behavior for higher $\tilde{n}$, We, $M$, and $N^{*}$. Table 3 displays variations in heat transfer rate against some parameters of interest. Tabulated values show that heat transfer rate decreases for Eckert number, Ec, dimensionless thermally stratified parameter, $S_{\theta}$, Brownian diffusion parameter, $N_{b}$, and 
Table 3. Numerical description of Nusselt number.

\begin{tabular}{ccccccc}
\hline $\mathbf{P r}$ & $\boldsymbol{R}_{\boldsymbol{d}}$ & $\mathbf{E c}$ & $\boldsymbol{S}_{\boldsymbol{\theta}}$ & $\boldsymbol{N}_{\boldsymbol{b}}$ & $\boldsymbol{N}_{\boldsymbol{t}}$ & $\mathbf{R e}_{\boldsymbol{x}}^{\frac{1}{2}} \mathbf{N u}_{\boldsymbol{x}}$ \\
\hline 1 & 0.5 & 1.6 & 0.3 & 0.1 & 0.1 & 0.2084664 \\
1.1 & & & & & & 0.2544226 \\
1.2 & & & & & & 0.3026473 \\
1 & 0.6 & 1.6 & 0.3 & 0.1 & 0.1 & 0.2492009 \\
& 0.7 & & & & & 0.2893210 \\
& 0.8 & & & & & 0.3293889 \\
1 & 0.5 & 1.7 & 0.3 & 0.1 & 0.1 & 0.2781875 \\
& & 1.8 & & & & 0.2288348 \\
& & 1.9 & & & & 0.1920219 \\
1 & 0.5 & 1.6 & 0.4 & 0.1 & 0.1 & 0.1800328 \\
& & & 0.5 & & & 0.1541903 \\
& & & 0.6 & & & 0.1291780 \\
1 & 0.5 & 1.6 & 0.3 & 0.2 & 0.1 & 0.1929118 \\
& & & & 0.3 & & 0.1779978 \\
& & & & 0.4 & & 0.1644246 \\
1 & 0.5 & 1.6 & 0.3 & 0.2 & 0.2 & 0.1849759 \\
& & & & & 0.3 & 0.1755373 \\
& & & & & 0.4 & 0.1683754 \\
\hline
\end{tabular}

Table 4. Numerical description of Sherwood number.

\begin{tabular}{ccccccc}
\hline $\mathbf{S c}$ & $\boldsymbol{A}^{\prime}$ & $\stackrel{\circ}{\boldsymbol{E}}$ & $\boldsymbol{S}_{\boldsymbol{\phi}}$ & $\boldsymbol{N}_{\boldsymbol{b}}$ & $\boldsymbol{N}_{\boldsymbol{t}}$ & $\mathbf{R e}_{\boldsymbol{x}}^{\frac{1}{2}} \mathbf{S h}_{\boldsymbol{x}}$ \\
\hline 0.8 & 0.1 & 1.1 & 0.1 & 0.2 & 0.3 & 0.3911616 \\
0.9 & & & & & & 0.4536701 \\
1 & & & & & & 0.5391772 \\
0.8 & 0.2 & 1.1 & 0.1 & 0.2 & 0.3 & 0.3758304 \\
& 0.3 & & & & & 0.3745837 \\
& 0.4 & & & & & 0.3651333 \\
0.8 & 0.1 & 1.2 & 0.1 & 0.2 & 0.3 & 0.3876376 \\
& & 1.3 & & & & 0.3654337 \\
& & 1.4 & & & & 0.3559579 \\
0.8 & 0.1 & 1.1 & 0.15 & 0.2 & 0.3 & 0.3536455 \\
& & & 0.2 & & & 0.3270567 \\
& & & 0.25 & & & 0.3119910 \\
0.8 & 0.1 & 1.1 & 0.1 & 0.25 & 0.3 & 0.5049517 \\
& & & & 0.3 & & 0.5660433 \\
& & & & 0.35 & & 0.6292429 \\
0.8 & 0.1 & 1.1 & 0.1 & 0.2 & 0.4 & 0.2766914 \\
& & & & & 0.5 & 0.1498125 \\
& & & & & 0.6 & 0.04072542 \\
\hline & & & & &
\end{tabular}

thermophoresis parameter, $N_{t}$, while it decreases for Prandtl number, Pr, and $R_{d}$. Further mass transfer rate is decreased via higher Schmidt number, Sc, and Brownian diffusion coefficient, $N_{b}$; however, it increases for the other parameters mentioned in Table 4.

\section{Conclusions}

Thermal and concentration stratifications under the effects of non-linear thermal radiation and activation energy were explored. The obtained results are presented in the following.

- Velocity field decays with larger Weissenberg number, We;

- Concentration distribution reduces with an increment in solutal stratification parameter, $S_{\phi}$;

- Temperature distribution significantly reduces for stronger thermal stratified parameter, $S_{\theta}$;

- Stronger chemical reaction parameter, $A^{\prime}$, results in the increase of concentration distribution;

- Velocity has a direct relation with mixed convection parameter, $\lambda$;

- Concentration against activation energy variable, $\stackrel{\circ}{E}$, increases;

- Heat transfer rate is enhanced for radiation parameter, $R_{d}$;

- Higher mass transfer rate is noted for thermophoresis parameter, $N_{t}$.

\section{References}

1. Cortell, R. "Fluid flow and radiative nonlinear heat transfer over a stretching sheet", J. King Saud. Univ. Sci., 26, pp. 161-167 (2014).

2. Kumar, R., Sood, S., and Sheikholeslami, M., et al. "Nonlinear thermal radiation and cubic autocatalysis chemical reaction effects on the flow of stretched nanofluid under rotational oscillations", J. Collo. Interf. Sci., 505, pp. 253-265 (2017).

3. Khan, M., Irfan, M., and Khan, W.A. "Impact of nonlinear thermal radiation and gyrotactic microorganisms on the Magneto-Burgers nanofluid", Int. J. Mech. Sci., 130, pp. 375-382 (2017).

4. Bhatti, M.M. and Rashidi, M.M. "Entropy generation with nonlinear thermal radiation in MHD boundary layer flow over a permeable shrinking/stretching sheet: Numerical solution", J. Nanofluids, 5, pp. 543-548 (2016).

5. Mustafa, M., Ahmad, R., Hayat, T., et al. "Rotating flow of viscoelastic fluid with nonlinear thermal radiation: a numerical study", Neural Comp. App., 29, pp. 493-499 (2018).

6. Hsiao, K.L. "Combined electrical MHD heat transfer thermal extrusion system using Maxwell fluid with radiative and viscous dissipation effects", Appl. Therm. Eng., 208 (2016). DOI: 10.1016/j.applthermaleng.

7. Hsiao, K.L. "Micropolar nanofluid flow with MHD and viscous dissipation effects towards a stretching sheet with multimedia feature", Int. J. Heat Mass Transf., 112, pp. 983-990 (2017). 
8. Turkyilmazoglu, M. "The analytical solution of mixed convection heat transfer and fluid flow of a MHD viscoelastic fluid over a permeable stretching surface", Int. J. Mech. Sci., 77, pp. 263-268 (2013).

9. Alamri, S.Z., Ellahi, R., Shehzad, N., et al. "Convective radiative plane Poiseuille flow of nanofluid through porous medium with slip: An application of Stefan blowing", J. Mol. Liq., 273, pp. 292-304 (2019).

10. Hassan, M., Marin, M., and Alsharif, A., et al. "Convection heat transfer flow of nanofluid in a porous medium over wavy surface", Physics Lett. A, 382, pp. 2749-2753 (2018).

11. Ellahi, R., Hassan, M., and Zeeshan, A. "Shape effects of spherical and nonspherical nanoparticles in mixed convection flow over a vertical stretching permeable sheet", J. Mech. Adv. Mat. Structures, 24, pp. 12311238 (2017).

12. Akbarzadeh, M., Rashidi, S., Karimi, N., et al. "Convection of heat and thermodynamic irreversibilities in two-phase, turbulent nanofluid flows in solar heaters by corrugated absorber plates", Adv. Powder Tech., 29, pp. 2243-2254 (2018).

13. Hsiao, K.L. "Stagnation electrical MHD nanofluid mixed convection with slip boundary on a stretching sheet", Appl. Therm. Eng., 98, pp. 850-861 (2016).

14. Hassan, M., Marin, M., Ellahi, R., et al. "Exploration of convective heat transfer and flow characteristics synthesis by $\mathrm{Cu}-\mathrm{Ag} /$ water hybrid-nanofluids", Heat Transf. Res., 49, pp. 1837-1848 (2018).

15. Hsiao, K.L. "To promote radiation electrical MHD activation energy thermal extrusion manufacturing system efficiency by using Carreau-nanofluid with parameters control method", Energy, 130, pp. 486-499 (2017).

16. Awad, F.G., Motsa, S., and Khumalo, M. "Heat and mass transfer in unsteady rotating fluid flow with binary chemical reaction and activation energy", Plos One, 9(9), e107622 (2014). https://doi.org/10.1371/journal.pone.0107622

17. Shafique, Z., Mustafa, M., and Mushtaq, A. "Boundary layer flow of Maxwell fluid in rotating frame with binary chemical reaction and activation energy", Results Phys., 6, pp. 627-633 (2016).

18. Mustafa, M., Khan, J.A., Hayat, T., et al. "Buoyancy effects on the MHD nanofluid flow past a vertical surface with chemical reaction and activation energy", Int. J. Heat and Mass Transf., 108, pp. 1340-1346 (2017).

19. Abbasi, F.M., Shehzad, S.A., Hayat, T., et al. "Mixed convection flow of Jeffrey nanofluid with thermal radiation and double stratification", J. Hydrodynamics, Ser. B, 28, pp. 840-849 (2016).
20. Daniel, Y.S., Aziz, Z.A., Ismail, Z., et al. "Effects of thermal radiation, viscous and Joule heating on electrical MHD nanofluid with double stratification", Chin. J. Phys., 55, pp. 630-651 (2017).

21. Ramzan, M., Bilal, M., and Chung, J.D. "Effects of thermal and solutal stratification on Jeffrey magnetonanofluid along an inclined stretching cylinder with thermal radiation and heat generation/absorption", Int. J. Mech. Sci., 132, pp. 317-324 (2017).

22. Ahmad, S., Farooq, M., Javed, M., et al. "Double stratification effects in chemically reactive squeezed Sutterby fluid flow with thermal radiation and mixed convection", Res. Phy., 8, pp. 1250-1259 (2018).

23. Liao, S.J., Homotopy Analysis Method in Non-linear Differential Equations, Springer and Higher Education Press, Heidelberg (2012).

24. Noeiaghdam, S., Zarei, E., and Kelishami, H.B. "Homotopy analysis transform method for solving Abel's integral equations of the first kind", Ain Shams Eng., J., 7, pp. 483-495 (2016).

25. Sui, J., Zheng, L., Zhang, X., et al. "Mixed convection heat transfer in power law fluids over a moving conveyor along an inclined plate", Int. J. Heat Mass Transfer, 85, pp. 1023-1033 (2015).

26. Turkyilmazoglu, M. "Solution of the Thomas-Fermi equation with a convergent approach", Commun. Nonlinear Sci. Numer. Simulat., 17, pp. 4097-4103 (2012).

27. Khan, M.I., Waqas, M., Hayat, T., Alsaedi, A., et al. "A comparative study of Casson fluid with homogeneous-heterogeneous reactions", J. Coll. Inter. Sci., 498, pp. 85-90 (2017).

28. Sheikholeslami, M., Ganji, D.D., Javed, M.Y., et al. "Effect of thermal radiation on magnetohydrodynamics nanofluid flow and heat transfer by means of two phase model", J. Magn. Magn. Mater., 374, pp. 36-43 (2015).

29. Hayat, T., Khan, M.I., Farooq, M., and Alsaedi, T., et al. "Impact of Cattaneo-Christov heat flux model in flow of variable thermal conductivity fluid over a variable thicked surface", Int. J. Heat Mass Transf., 99, pp. 702-710 (2016).

30. Hayat, T., Qayyum, S., and Alsaedi, A., et al. "Nonlinear thermal radiation aspects in stagnation point flow of tangent hyperbolic nanofluid with double diffusive convection", J. Mol. Liq., 223, pp. 969-978 (2016).

31. Hayat, T., Sajid, M., and Pop, I. "Three-dimensional flow over a stretching surface in a viscoelastic fluid", Nonlinear Analysis: Real World Appl., 9, pp. 18111822 (2008).

32. Abbasbandy, S. and Hayat, T. "Solution of the MHD Falkner-Skan flow by homotopy analysis method", Comm. Nonlinear Sci. Numer. Simulation, 14, pp. 3591-3598 (2009). 
33. Hayat, T., Waqas, M., Shehzad, S.A., et al. "Effects of Joule heating and thermophoresis on the stretched flow with convective boundary condition", Scientia Iranica, 21, pp. 682-692 (2014).

34. Eswaramoorthi, S., Sivanandam, S., Bhuvaneswari, M., et al. "Soret and Dufour effects on viscoelastic boundary layer flow over a stretching surface with convective boundary condition with radiation and chemical reaction", Scientia Iranica, 23, pp. 2575-2586 (2016).

35. Qayyum, S., Hayat, T., Shehzad, S.A., et al. "Mixed convection and heat generation/absorption aspects in MHD flow of tangent hyperbolic nanoliquid with Newtonian heat/mass transfer", Rad. Phy. Chem., 144, pp. 396-404 (2018).

\section{Biographies}

Sumaira Jabeen is a PhD student of Mathematics at Quaid-i-Azam university, Pakistan. She received her master's degree from Quaid-i-Azam University. Her research interests include fluid mechanics, non-linear flow problems, and heat transfer.

Tasawar Hayat is a Pakistani Mathematician who has made pioneering research contributions to the area of mathematical fluid mechanics. He is considered one of the leading mathematicians working in Pakistan and, currently, is a Professor of Mathematics at the Quaidi-Azam University.

Ahmad Alsaedi is a Professor at the Department of Mathematics at King Abdulaziz University, Jeddah, Saudi Arabia. He is a member of Nonlinear Analysis and Applied Mathematics (NAAM) research group. His areas of interest include fluid dynamics, nonlinear flow analysis, and flow problem in nanosystems.

Mohammed Sh Alhodalay is an Assistant Professor at King Abdulaziz University. His research interests are fluid dynamics, heat transfer, and combustion. 